\title{
Implementation of a combined Cardiopulmonary Resuscitation and Treatment Escalation Plan document in a District General Hospital
}

\author{
Claire Stockdale, Bhavi Trivedi, Ellen Jerome, Samir Salih, Christopher Huntley, Eleanor Cooke, Yolanda Massey, Sophie Mella \\ Musgrove Park Hospital, Taunton
}

\begin{abstract}
Documentation of appropriate escalation of treatment was identified as a problem for junior doctors and Critical Care Outreach Nurses at Musgrove Park Hospital. An audit of resuscitation and escalation documentation of all wards found that of the patients who were not for Cardiopulmonary resuscitation (and therefore not for full escalation of care), $78.4 \%$ had no documentation of the appropriate level of escalation of treatment should they deteriorate. The majority of junior doctors had experienced cases where they felt that inappropriate treatment had been given, where no escalation plan was documented.
\end{abstract}

Using several Plan, Do, Study, Act (PDSA) cycles, drawing tools used in other trusts and departments, and the views of clinicians, we developed a treatment escalation plan (TEP) tool, to be included in the resuscitation form. This included consideration of referral to critical care, ward based non-invasive ventilation, and appropriate use of intravenous or oral antibiotics. This then prompted the responsible clinician to consider and document appropriate escalation of treatment.

The CPR-TEP form was trialed using a quasi-experiment design allowing the aim to be tested using two groups - intervention and control. All patients in the intervention group were not for CPR and therefore had their TEP-CPR form filled in fully $(n=68)$. The control group consisted of patients who were not for CPR but who did not have a TEP form filled in $(n=36)$.

The appropriateness of $\mathrm{OOH}$ (out of hours) treatment in those patients who experienced clinical deterioration was judged by questionnairebased feedback from the in-hours team the following morning. Levels of inappropriate treatment between the two groups were compared to test the aim.

At the end of the study period, questionnaire feedback indicated that $11.1 \%$ of patients in the group with the new CPR-TEP document had received inappropriate $\mathrm{OOH}$ care compared to $44.4 \%$ of patients in the group without the document.

Using the TEP alongside resuscitation documentation prompts the responsible clinician to consistently consider and document the appropriate escalation of care for their patient, improving communication with the out of hours team and appropriate escalation of care in the event of patient deterioration.

\section{Problem}

For patients in an acute hospital setting, deterioration in clinical condition frequently occurs outside of normal working hours. In some patients, cardio-pulmonary resuscitation (CPR) is unlikely to improve survival but this does not preclude the use of other resuscitative treatment modalities, such as intravenous fluids and antibiotics, or more invasive measures like ventilator support and inotrope/vasopressor therapy. Patient factors, including pre-morbid function, influence the clinical benefit conferred by individual treatment modalities, but any pre-existing wishes and values will also help determine which of these measures may be inappropriate. Within working hours there is greater opportunity to explore these factors, and often it is possible for in-hours teams to identify patients at foreseeable risk of deterioration. In these patients, documentation of any inappropriate treatments allows the in-hours team to tailor care to the individual's clinical circumstances and guide out-of-hours $(\mathrm{OOH})$ management, thus promoting beneficence. Our preliminary research suggested that the resuscitative treatment modalities used $\mathrm{OOH}$ in patients categorised 'not for CPR', was sometimes considered by in-hours teams to conflict with best interests.

\section{Background}

The principle of beneficence dictates that the health professional's duty is to act in the best interests of the patient at all times. Prolonging life at all costs without due regard to the burden of treatment conflicts with this principle, and it is thus the duty of the doctor to carefully consider the likelihood of benefit from each treatment modality, on an individual patient basis, giving thought to pre-morbid level of function, beliefs and wishes. Blanket policies of 'for all treatment' or 'for no treatment' are ethically indefensible.

The resuscitation council outlined that ;advance care planning, including making decisions about CPR, is an important part of good clinical care for those at risk of cardio-pulmonary arrest.' However, other trusts have already identified that in patients unlikely to benefit 
from CPR, quality of care can be improved by documenting a treatment escalation plan (TEP) to guide management in the event of an $\mathrm{OOH}$ clinical deterioration. By tailoring management to the individual patient's clinical circumstances and subsequently minimising the risk of on-call teams issuing treatments with limited conferred benefit, TEPs can promote beneficence. On a TEP, all available treatment modalities are laid out individually, with clear indication beside those which may be inappropriate; these decisions are dynamic and can change with variation in clinical condition. They should ideally be discussed with the patient early in their illness while capacity to make specific decisions about future life sustaining treatments is still preserved. If the patient lacks capacity to participate in decision making, a Lasting Power of Attorney (where one exists) and the family and next of kin should be engaged.

The aim of the study was to identify whether creating a TEP within normal working hours in patients categorised 'not for CPR' could reduce the number of patients receiving inappropriate treatments during $\mathrm{OOH}$ deterioration. This was in comparison to similarly categorised patients who had no written documentation of inappropriate treatment modalities.

\section{Baseline Measurement}

On a single day, the clinical notes of 178 patients were audited. In those who had a completed CPR form (58\%; $n=103), 46 \%(n=81)$ were 'not for CPR' but in more than three quarters of these patients ( $78 \% ; n=61)$, there was no documentation of which treatment modalities were considered to be inappropriate in the event of clinical deterioration. On the same day, the number of patients who experienced an $\mathrm{OOH}$ clinical deterioration (5pm-9am) requiring review by on-call doctors was recorded $(n=30)$. In $40 \%(n=12)$ and no CPR decision was documented. In the remainder $(n=18)$ with a CPR decision, 'not for CPR' was documented in 50\% ( $n=9)$ but $77 \%$ of these patients $(n=7)$ had no written documentation of treatment measures considered inappropriate. In the two patients who did have a documented ceiling of escalation, the phrase 'in the event of deterioration, this patient is for ward based care only' was used, with no mention of specific resuscitative treatment options. Subsequent feedback from the in-hours team regarding appropriateness of the $\mathrm{OOH}$ treatment in these 9 patients was assessed via questionnaire the following morning. In $44 \%(n=4)$, one or more treatment modalities were felt to have been inappropriate for the patient in question and not in their best interests.

In a survey of junior doctors in the hospital, $86 \%$ recalled at least one occasion where they had required a senior colleague to complete a CPR form after deterioration in a patient's clinical condition. All of the doctors surveyed felt that a treatment escalation plan documented by the in-hours team would have been useful to guide management $\mathrm{OOH}$.

\section{Design}

Failure to document any inappropriate treatment modalities for individual patients stems partly from reluctance to consider clinical deterioration before it has happened. The situations in which patients deteriorate can be complex and the appropriateness of treatments may differ based on the circumstances surrounding the deterioration. By adapting a TEP document already established in another NHS trust and incorporating it into our trust's existing CPR form, the new TEP-CPR form aimed to force clinicians to consider both the possibility of deterioration and the appropriateness of individual treatment modalities when making decisions about CPR. The basic structure of the original CPR form was maintained because improved participation in a trial of a form-based intervention has been previously documented when the intervention is introduced into a familiar design

A quasi-experiment design was used to test the aim in two groups intervention and control. All patients in the intervention group had a fully completed TEP-CPR form; the TEP component was only completed if the CPR decision was 'not for CPR' thus everyone in the intervention group was 'not for CPR'. The control group included all the patients on the remaining adult medical and surgical wards who were 'not for CPR' but had no documentation of what treatments were considered inappropriate in the event of clinical deterioration. Thus the independent variable was whether or not a patient had a new CPR-TEP document. The dependent variable was appropriateness of $\mathrm{OOH}$ treatment in those patients who experienced clinical deterioration $\mathrm{OOH}$, as judged by questionnairebased feedback from the in-hours team the following morning. Levels of inappropriate treatment between the two groups were compared to test the aim.

\section{Strategy}

In total, four PDSA cycles were used to develop the final TEP-CPR form.

PDSA 1 - the new TEP-CPR form was shown to several elderly care and oncology consultants. In elderly patients and in those with cancer, there is often a foreseeable risk of clinical deterioration. The advice of consultants in these fields was thus sought because they frequently engage in decision making about what treatments would be in an individual's best interests in the event of clinical deterioration. Initially six resuscitative treatment modalities were laid out in a pyramid design with simple measures ( IV fluids, antibiotics and blood transfusions) placed lower in the pyramid and more invasive measures (inotrope/vasopressor therapy, renal replacement therapy and invasive ventilation) placed higher. The aesthetics of the design were well received however there was concern that decision making regarding the more invasive treatments would be difficult for clinicians as the appropriateness of each may depend on the specific clinical condition. Since all three invasive measures are facilitated in the HDU/ITU setting, they were merged under one unifying option 'for escalation to HDU/ITU'. The resuscitative treatment options on the modified TEP-CPR from, in order of least invasive (bottom of the pyramid) to most invasive (top of the pyramid) was thus IV fluids, antibiotics and escalation to HDU/ITU.

PDSA 2- the modified TEP-CPR was piloted for 2 weeks on an 
oncology ward and an elderly care ward. These wards were specifically chosen because they had the highest rates of $\mathrm{OOH}$ clinical deterioration. In this initial stage, usability of the form, factors influencing completion and safety of the form were assessed. Although there was unanimous agreement that the form was user-friendly, concerns over the safety of the form were raised. There is huge variability in the circumstances under which a patient's clinical condition may deteriorate. In the same patient, treatments which are judged inappropriate may in other instances be considered appropriate. Thus in the event of a patient surviving a deterioration, there was a theoretical risk of the TEP-CPR form displaying advice that may not be valid for a further deterioration under different circumstances. Many consultants were uncomfortable with this idea from a legal and professional point of view. Mandatory documentation of a date for review of the decisions was suggested by some. A series of additional boxes were also included to indicate to the $\mathrm{OOH}$ team that the designated review had taken place and advise them of when the in-hours team felt the next review was necessary. The absence of evidence that the form had been reviewed would invalidate the advice suggested by the in-hours team; in this situation the patient was viewed as if they had no TEP-CPR form. If there was no evidence in one of the provided boxes that this review had occurred, the decisions on the form would be invalidated. Other situations rendering the form invalid were discharge from hospital and transfer to another healthcare setting. The responsibility for completion and review of the TEP-CPR form rested with the most senior clinician in charge of the patient's care, but it was possible for the task to be delegated to another member of the team provided level of training was greater than ST3 (specialty trainee year 3). It was advised that wherever possible, the patient should be involved in decision making and that ideally the decisions should be agreed with the whole healthcare team.

PDSA 3 - following the initial pilot and above modifications to the TEP-CPR form, a further one week pilot was performed on the same wards using the most up to date version of the form. The addition of the special circumstances box was well received and allowed clinicians to be less rigid in their decision making, which had previously been cited as a criticism from several consultants. Feedback from the second pilot indicated that the mandatory review date and provision of additional boxes to indicate time of next review, eased the anxiety surrounding use of the previous form. No further suggestions were made and the final version of the form was thus created.

PDSA 4 - The form was used for a further 2 months on the oncology ward and the elderly care ward. The TEP component was completed by the clinical team within working hours in all patients who were deemed to be 'not for CPR'. The intervention group was all of the patients on these 2 wards with TEP component of the form completed, who experienced clinical deterioration $\mathrm{OOH}$.

Questionnaire-based feedback from the in-hours team about the appropriateness of the $\mathrm{OOH}$ treatment given, was collected the following morning for these patients. Over the study period, the difference in appropriateness of $\mathrm{OOH}$ treatment with use of the TEPCPR form was compared to treatment of patients in the control group who deteriorated $\mathrm{OOH}$. The control group included patients on other hospital wards with a completed CPR form documenting 'not for CPR' but without any documentation of TEP in the notes.

\section{Results}

The number of admissions to the two intervention wards over the study period was 90; the TEP component of the new document was completed in $75.5 \%$ ( $n=68)$, for all patients whose CPR decision was 'not for CPR'. In this group, $66 \%(n=45)$ had an $\mathrm{OOH}$ clinical deterioration during the study period. There were 36 patients in the comparison group. At the end of the study period, questionnaire feedback indicated that $11.1 \%$ of patients in the group with the new CPR-TEP document had received inappropriate $\mathrm{OOH}$ care compared to $44.4 \%$ of patients in the group without the document). Analysis of the medical notes in the 5 patients in the intervention group whose $\mathrm{OOH}$ care was deemed inappropriate despite a completed CPR-TEP form identified that the documented ceiling of appropriate treatment modalities had not been followed due to disagreement by the medical registrar on call.

\section{Lessons and Limitations}

The limitations of the study are related to the study design. Patients were not assigned randomly to the intervention group. The CPRTEP document was completed for patients on the two wards in the hospital with the highest rates of $\mathrm{OOH}$ clinical deterioration. These wards were an acute elderly care ward and an oncology ward. In these patients there was foreseeable risk of clinical deterioration and due to the high proportion of time that is spent communicating with patients and relatives/carers on these wards, a great deal is known about the patient's wishes thus it may have been easier to decide which treatment modalities would be in the patient's best interests. For patients on the other wards in whom CPR was deemed futile but without obvious potential for clinical deterioration, deciding which treatment modalities are in the patient's best interests may not be so easy; what is or is not appropriate may depend on the nature of deterioration. Our sample size was very small and although our data is promising, it is limited in its power. To improve this we need to monitor greater numbers of patients who have deteriorated and then follow up with feedback from inhours teams.

\section{Conclusion}

In-hours clinical teams have greater opportunity to explore patients' pre-morbid function, wishes and values by talking directly to the patient as well as relatives/carers. They are therefore better placed to make decisions about which treatment modalities are in the best interests of the patient, in the event of clinical deterioration. Despite this, documentation of these decisions is poor. Most clinical deterioration occurs $\mathrm{OOH}$ and when these important decisions are left solely to on-call teams, the management can be subsequently viewed by the in-hours team to be conflicting with the best interests of the patient. This study has shown that this disparity can be reduced by completing a TEP in patients who, despite perceived futility of $\mathrm{CPR}$, may require other resuscitative treatment modalities $\mathrm{OOH}$. TEPs can therefore promote beneficence. 
BMJ Quality Improvement Reports

The proportion of patients dying in the setting of an acute hospital ward (about 60\%) does not correlate with data from public surveys about preferred place of dying. As many as two thirds of patients reaching the end of life would prefer to die at home or in a hospice environment and the Second Annual Report of the End of Life Care Strategy suggests that many people are needlessly sent to hospital to die simply because care home staff are unsure of what else to do (1). Every effort should be made to address this inconsistency particularly in view of the fact that $54 \%$ of complaints in acute hospitals are related to care of the dying, according to national survey undertaken by the Health Care Commission in 2007. Indeed the NHS End of Life Care Strategy stipulates that all people approaching the end of life should have their needs assessed, their wishes and preferences discussed and an agreed set of actions reflecting the choices they make about their care recorded in a care plan (2). Historically, this aim has not been well achieved. Success has largely been limited by the attitude - endemic within our society - that death and dying is a taboo area. It is imperative to raise the profile of end of life care in order to change this negative attitude both within the medical community and among the public.

Considering any treatment modalities which may be inappropriate for the individual patient with subsequent written documentation in the form of a TEP is just one small step towards achieving this cultural change. Ideally, advance care planning (ACP) should be at the forefront of the clinicians mind, whether this is in an outpatient clinic or GP practice. However, identifying groups of patients who might benefit from ACP and ensuring that the clinician has enough time for these discussions can be difficult. One way to prompt clinicians to consider ACP is to use the Gold Standards Framework Surprise Question, "would you be surprised if this patient died in the next 12 months". If the answer to the question is 'no' a conversation about the patient's beliefs and wishes on potential life sustaining treatment should be initiated and documented in the medical notes. It is important to do this while the patient has capacity to make these decisions, which may be lost in the event of future deterioration. Research suggests that the outpatient clinic setting is an ideal place to discuss these important issues as there is greater privacy than on the open hospital ward and patients are often seen over a long period of time by the same consultant (or his/her team).

All doctors will at some point be involved in end of life care and should therefore take an interest in ensuring that quality of life during this period.

\section{References}

1. NHS End of Life Care Strategy. Promoting high quality care for all adults at the end of life. Department of Health, London. July 2008; Page 12, section 14.

2. The Second Annual Report of the End of Life Care Strategy, Department of Health. London, August 2010.

National End of Life Care Programme. The route to success. Department of Health. London, 2010.

\section{Declaration of interests}

None

\section{Acknowledgements}

Dr Peter Cavanagh, Dr Peter Campbell, Dr Lucy Pollock, Dr Julie Walthers, Dr Joanne Watson 